\title{
Low-frequency electroacupncture improves locomotor function after sciatic crushed nerve injury in rats
}

\author{
Key-Moon Shin', II-Gyu Ko', Sung-Eun Kim¹, Jun-Jang Jin', Lakkyong Hwang'1, Sang-Hoon Kim², , Jin-Hee Seo³, Bo-Kyun Kim", \\ Yong Gil Na ${ }^{5, *}$ \\ 'Department of Physiology, College of Medicine, Kyung Hee University, Seoul, Korea \\ ${ }^{2}$ Department of Sport \& Health Science, College of Natural Science, Sangmyung University, Seoul, Korea \\ ${ }^{3}$ Department of Adaptive Physical Education, Baekseok University, Cheonan, Korea \\ ${ }^{4}$ Department of Emergency Technology, College of Health Science, Gachon University, Incheon, Korea \\ ${ }^{5}$ Department of Urology, Chungnam National University Hospital, Chungnam National University College of Medicine, Daejeon, Korea
}

Sciatic crushed nerve injury (SCI) causes pain-related gait and swelling in the affected limb. Electroacupuncture (EA) is a modified acupuncture technique, and analgesic effect of EA on different types of pain has been documented. Scientific functional index (SFI) is a mathematical formula to represent parameters of normal and experimental footprints. We investigated the effect of low-frequency EA on functional recovery following SCl in rats. For this study, immunohistochemistry for c-Fos in the ventral lateral periaqueductal gray (vIPAG) and paraventricular nucleus (PVN) and western blot for neurofilament (NF) and brain-derived neurotrophic factor (BDNF) in the sciatic nerve were conducted. To induce crush injury on the sciatic nerve, sciatic nerve was crushed for 30 sec using a surgical clip. The rats in the acupuncture groups received acupuncture bilaterally at respective site, once a day for 14 days. The rats in the EA group received $100-\mathrm{Hz}$ electrical stimulation for $10 \mathrm{~min}$ once a day during 14 days. SCI decreased SFI value, in contrast, EA increased SFI value. C-Fos expression in the vIPAG and PVN was increased following $\mathrm{SCl}$, in contrast, EA suppressed c-Fos expression. NF expression in the sciatic nerve was decreased by $\mathrm{SCl}$, in contrast, $\mathrm{EA}$ increased NF expression. BDNF expression in the sciatic nerve was increased by $\mathrm{SCl}$, in contrast, EA suppressed BDNF expression. In the present study, EA showed effectiveness on functional recovery from SCI.

Keywords: Electroacupuncture, Scientific functional index, c-Fos, Neurofilament, Brain-derived neurotrophic factor

\section{INTRODUCTION}

Peripheral nerves are often damaged by crush, compression, stretching, avulsion, and division. The injury of nerve causes loss of function of internal organs and muscles. Following sciatic crushed nerve injury (SCI), pain-related gait and swelling are displayed in the affected limb.

Ascending central pathways transmit nociceptive information to the pain-related brain areas (Price, 2000). Of these, periaqueductal gray (PAG) and paraventricular nucleus (PVN) are important brain sites involved in ascending pain transmission. It receives afferent from nociceptive neurons in the spinal cord and send pro- jection to the thalamic nuclei that process nociception.

Immediate early gene $c$-fos has been considered as a marker for stimuli-induced metabolic change of neurons. Expression of c-Fos is enhanced by various stimuli and c-Fos is a sensitive marker for the neural activation (de Medeiros et al., 2003; Narita et al., 2003). c-Fos expression in the PAG and PVN suggests that neuronal activation occurs in a location-specific manner (Belevych et al., 2010).

Neurofilament (NF) is an intermediate filament that found in neurons. NF is a major component of the neuronal cytoskeleton, and provides structural support for the axon (Park et al., 2013). NF-200 is one of the types of cytoskeletal protein and NF-200
${ }^{*}$ Corresponding author: Yong Gil Na (D) https://orcid.org/0000-0002-0794-5459 Department of Urology, Chungnam National University Hospital, Chungnam National University College of Medicine, 266 Munhwa-ro, Jung-gu, Daejeon 35015, Korea

Tel: +82-42-280-7778, Fax: +82-42-221-1075, E-mail: yongna@cnu.ac.kr Received: October 15, 2018 / Accepted: November 26, 2018
This is an Open Access article distributed under the terms of the Creative Commons Attribution Non-Commercial License (http://creativecommons.org/licenses/by-nc/4.0/) which permits unrestricted non-commercial use, distribution, and reproduction in any medium, provided the original work is properly cited. 
plays a role in the stabilization and maturation of pre-existing connections (Kriz et al., 2000).

Brain-derived neurotrophic factor (BDNF) is a member of the neurotrophic family and BDNF promotes the survival of neurons during development. BDNF modulates many morphological changes such as dendritic ar $\neg$ borization, remodeling of axon and dendrite, and synaptogenesis (Sallert et al., 2009; Yacoubian and Lo, 2000). BDNF up-regulation represents neuronal regeneration and improvement of locomotor function (Jung et al., 2016; Kim et al., 2017).

The scientific functional index (SFI) is a mathematical formula to represent parameters of normal and experimental footprints. SFI provides information of gait function associated with sensory-motor connections (de Medinaceli et al., 1982). SFI has been revised several times to become a simpler and more reliable index. SFI is used to assess functional improvement after SCI (Byun et al., 2005; Lee et al., 2018).

Acupuncture has been used for to treat various maladies, especially pain (Cherkin et al., 2003). Electroacupuncture (EA) is a modified acupuncture technique, as its name implies, that utilizes electrical stimulation. Analgesic effect of EA on different types of acute pain and persistent inflammatory pain has been documented in rodents and humans (Baek et al., 2005; Chang et al., 2004; Gim et al., 2011). Zusanli acupoint (ST36) is the most commonly used acupoint for the pain control and immune regulation (Chang et al., 2004). EA relieves the behavioral signs of hyperalgesia and allodynia associated with neuropathic pain (Dong et al., 2006; Huang et al., 2004; Gim et al., 2011).

In the present study, we investigated the effect of low-frequency EA on functional recovery following SCI in rats. For this study, immunohistochemistry for c-Fos in the ventral lateral PAG (vlPAG) and PVN and western blot for NF-200 and BDNF in the sciatic nerve were conducted.

\section{MATERIALS AND METHODS}

\section{Animals and treatments}

Male Sprague-Dawley rats weighing $220 \pm 10 \mathrm{~g}$ (8 weeks old) were used and the experiment was performed in accordance with the animal care guidelines of the National Institute of Health and the Korean Academy of Medical Sciences. The rats were randomly divided into five groups ( $\mathrm{n}=10$ in each group): the sham-operation group, the SCI-induced group, the SCI-induced and nonacupoint acupuncture group, the SCI-induced and ST36-acupoint acupuncture group, the SCI-induced and EA group.
The rats in the acupuncture groups received acupuncture bilaterally at respective site, once a day for 14 days. For acupunctural stimulation, after insertion of stainless acupuncture needles $(0.3$ $\mathrm{mm}$ ), the needles were manually rotated clockwise and counter-clockwise 30 times and then kept in place for $10 \mathrm{~min}$. Nonacupoint is located at the side of the hip. ST36-acupoint (Zusanli) is located at the four fingers below the tibialis anterior muscle. The rats in the EA group received $100-\mathrm{Hz}$ electrical stimulation for 10 min once a day during 14 days.

\section{Crush injury on sciatic nerve}

To induce crush injury on the sciatic nerve, the previously described surgical procedure was performed (Byun et al., 2005; Lee et al., 2018). Right sciatic nerve was exposed by incision on the gluteal muscle under anesthesia using Zoletil $50(50 \mathrm{mg} / \mathrm{kg}$, intraperitoneally; Vibac Laboratories, Carros, France). The sciatic nerve was carefully exposed and crushed for 30 sec using a surgical clip (pressure: 125 g; Fine Science Tool Inc., San Francisco, CA, USA). The crushed location was between the sciatic notch and the point of trifurcation.

\section{Walking tract analysis}

Functional recovery after SCI was quantified by SFI. Examination of the walking patterns was performed 3 times with 1 day intervals through the course of the experiment as the previously described method (Byun et al., 2005; Lee et al., 2018). Foot-prints were recorded in a wooden walking alley $(8.2 \mathrm{~cm} \times 42 \mathrm{~cm})$ with a darkened goal box at the end. The floor of the alley was covered with white paper. The anatomical landmarks on the hind feet of the rats were smeared with finger point. The rat was allowed to walk down the tract, leaving its foot-prints on the paper.

Following parameter were calculated from the footprints: distance from the heel to the top of the 3rd toe (print length, PL), distance between the 1st to the 5 th toe (toe spread, TS), and distance from the 2nd to the 4th toe (intermediary toe spread, IT). These parameters were taken both from the intact left (non-operated) foot (NPL, NTS, and NIT) and from the injured right (experimental) foot (EPL, ETS, and EIT). SFI values were obtained using following equation: $\mathrm{SFI}=(-38.3 \pm \mathrm{PLF})+(109.5 \pm \mathrm{TSF})+$ $(13.3 \pm$ ITF $)-8.8$. Print length factor $(\mathrm{PLF})=(\mathrm{EPL}-\mathrm{NPL}) / \mathrm{NPL}$; toe spread factor $($ TSF $)=($ ETS-NTS $) /$ NTS; intermediary toe spread factor $($ ITF $)=($ EIT-NIT $) /$ NIT. Interpolating identical values of PL, TS, and IT from the right and left hind feet are close to zero in normal rats. A value of -100 indicates complete impairment. 


\section{Tissue preparation}

The rats were sacrificed immediately after last walking tract analysis (15 days after inducing of SCI). After fully anesthetizing the rats using Zoletil $50(10 \mathrm{mg} / \mathrm{kg}$, intraperitoneally; Vibac Laboratories), $50 \mathrm{mM}$ phosphate-buffered saline (PBS) was transcardially perfused, and then fixed with $4 \%$ paraformaldehyde in 100 $\mathrm{mM}$ phosphate buffer at $\mathrm{pH} 7.4$. After dissecting brains, the brains were postfixed in the same fixative overnight, and transferred to $30 \%$ sucrose for cryoprotection. Coronal sections of $40-\mu \mathrm{m}$ thickness were made with a freezing microtome (Leica, Nussloch, Germany). The sections were finally mounted onto gelatin-coated slides. The slides were air-dried overnight at room temperature, and the coverslips were mounted using Permount (Fisher Scientific, Fair Lawn, NJ, USA).

\section{Immunohistochemistry for c-Fos}

For immunolabeling of c-Fos in the PVN and vlPAG, c-Fos immunohistochemistry was performed as the previously described method (Han et al., 2017; Ko et al., 2016). Free-floating tissue sections were incubated overnight with rabbit anti-c-Fos antibody (1:1,000; Santa Cruz Biotechnology, Santa Cruz, CA, USA), and the section were then incubated for $1 \mathrm{hr}$ with biotinylated antirabbit secondary antibody (1:200; Vector Laboratories, Burlingame, CA, USA). The sections were subsequently incubated with avidinbiotin-peroxidase complex (1:100; Vector Laboratories) for $1 \mathrm{hr}$ at room temperature. Immunoreactivity was visualized by incubating the sections in a solution consisting of $0.05 \% 3,3$-diaminobenzidine and $0.01 \% \mathrm{H}_{2} \mathrm{O}_{2}$ in $50 \mathrm{mM}$ Tris-buffer $(\mathrm{pH}, 7.6)$ for approximately $3 \mathrm{~min}$. After washing three times with PBS, the section was mounted onto gelatin-coated slides. At room temperature, the slides were air-dried overnight, and coverslips were mounted using Permount (Fisher Scientific).

\section{Western blot for NF-200 and BDNF}

Western blot analysis for the NF-200 and BDNF was performed as the previously described method (Jung et al., 2016; Kim et al., 2017; Park et al., 2017). The sciatic nerve tissues were collected, and then were immediately frozen at $-70^{\circ} \mathrm{C}$. After homogenizing sciatic nerve tissues, tissue were lysed in a lysis buffer containing $50 \mathrm{mM}$ HEPES (pH, 7.5), $150 \mathrm{mM} \mathrm{NaCl}, 10 \%$ glycerol, $1 \%$ Triton X-100, 1 mM PMSF, $1 \mathrm{mM}$ EGTA, $1.5 \mathrm{mM} \mathrm{MgCl} 2 \cdot 6 \mathrm{H}_{2} \mathrm{O}, 1$ $\mathrm{mM}$ sodium orthovanadate, and $100 \mathrm{mM}$ sodium flouride. BioRad colorimetric protein assay kit (Bio-Rad, Hercules, CA, USA) was used to determine protein content. Protein $(30 \mu \mathrm{g})$ was separated on sodium dodecyl sulfate-polyacrylamide gels and trans- ferred onto a nitrocellulose membrane.

For the primary antibody, mouse beta-actin antibody $(1: 1,000$; Santa Cruz Biotechnology), mouse NF-200 antibody (1:1,000; Santa Cruz Biotechnology), and rabbit BDNF antibody (1:1,000; Santa Cruz Biotechnology) were used. As the secondary antibody, horseradish peroxidase-conjugated anti-mouse antibody (1:3,000; Amersham Pharmacia Biotechnology GmbH, Freiburg, Germa-

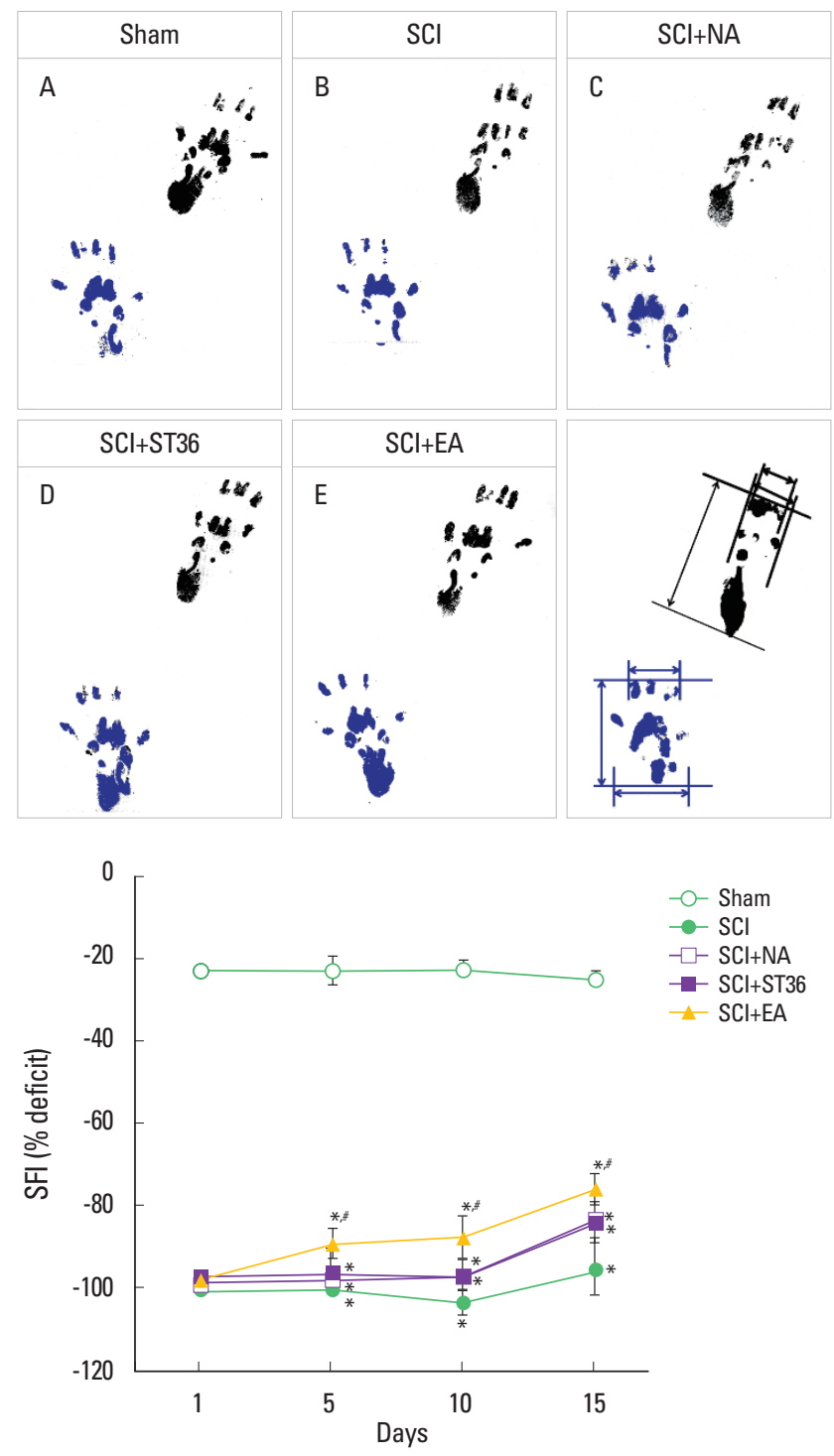

Fig. 1. Effect of electric stimulation (ES) on sciatic functional index (SFI). Upper panel: Walking tract foot-print in sciatic crushed nerve injury (SCI). A, sham-operation group; B SCl-induced group; C, SCl-induced and nonacupoint acupuncture group; D, SCl-induced and ST36-acupoint acupuncture group; E, SCl-induced and electroacupuncture (EA) group. Lower panel: SFI following sciatic nerve injury. NA, nonacupoint acupuncture; ST36, ST36-acupoint acupuncture. ${ }^{*} P<0.05$ compared to the sham-operation group. ${ }^{\sharp} P<0.05$ compared to the SCl-induced group. 
ny) for beat-actin and NF-200, and anti-rabbit antibody (1:2,000; Vector Laboratories) for BDNF were used. Enhanced chemiluminescence detection kit (Santa Cruz Biotechnology) was used for the band detection.

\section{Data analysis}

The areas of PVN and vlPAG from each slice were measured using Image-Pro Plus computer-assisted image analysis system (Media Cybernetics Inc., Bethesda, MD, USA) with to a light microscope (Olympus, Tokyo, Japan). The numbers of c-Fos-positive cells in the PVN and vlPAG were counted hemilaterally through a light microscope (Olympus). To compare the relative expressions of NF-200 and BDN, the detected bands were calculated densitometrically using Image-Pro Plus software (Media Cybernetics Inc.).

Statistical analysis was performed using one-way analysis of
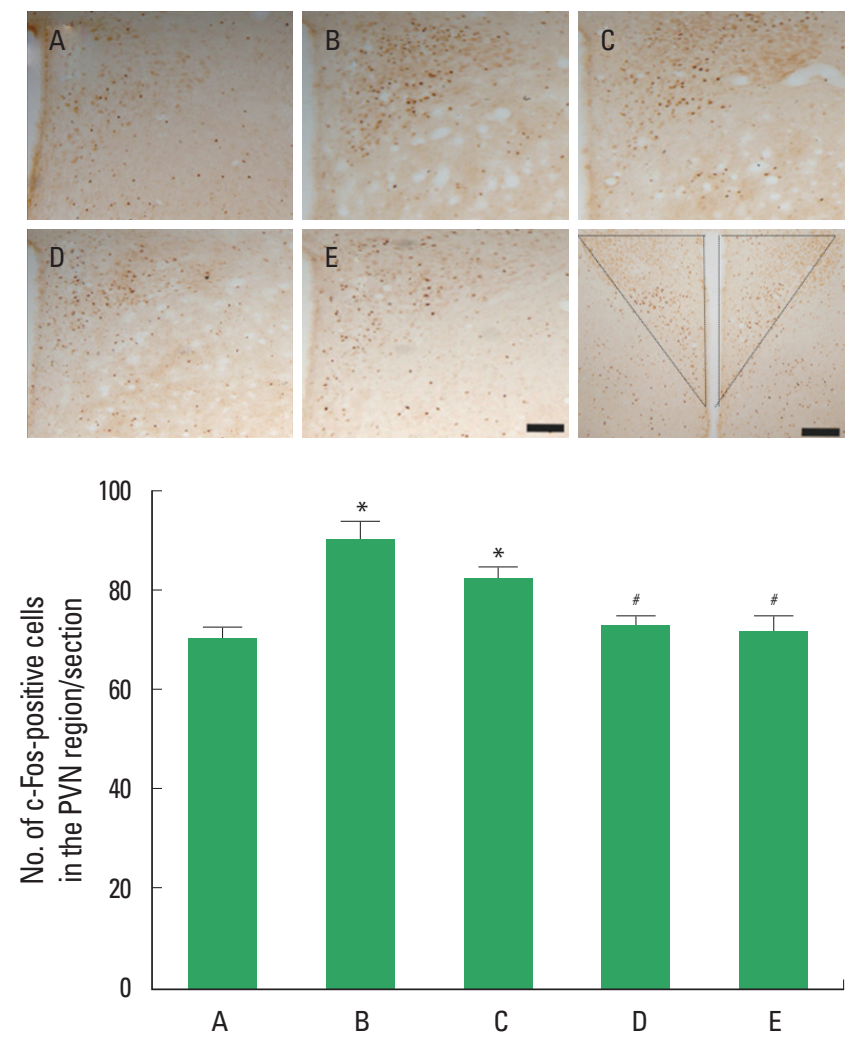

Fig. 2. Effect of electroacupuncture on c-Fos expression in the paraventricular nucleus (PVN). Upper panel: Photomicrographs showing immunostaining for c-Fos-positive cells. The scale bar represents $100 \mu \mathrm{m}(\mathrm{A}-\mathrm{E})$ and $400 \mu \mathrm{m}$. Lower panel: Number of c-Fos positive cells in each group. $A$, sham-operation group; $\mathrm{B}$, sciatic crushed nerve injury (SCI)-induced group; C, SCl-induced and non-acupoint acupuncture group; D, SCl-induced and ST36-acupoint acupuncture group; $\mathrm{E}, \mathrm{SCl}$-induced and electroacupuncture group. ${ }^{*} P<0.05$ compared to the sham-operation group. ${ }^{\#} P<0.05$ compared to the $S C l$-induced group. variance followed by Duncan post hoc test. The results are presented as the mean \pm standard error of the mean. Significance was set as $P<0.05$.

\section{RESULTS}

\section{SFI following SCI}

Foot-prints are presented in Fig. 1. The present results showed that the SFI was decreased by induction of SCI. Acupuncture and EA treatment enhanced the SFI. EA showed most potent increasing effect on SFI.

\section{c-Fos expression in the PVN}

c-Fos expressions in the PVN are presented in Fig. 2. The present results showed that the number of c-Fos-positive cell in the
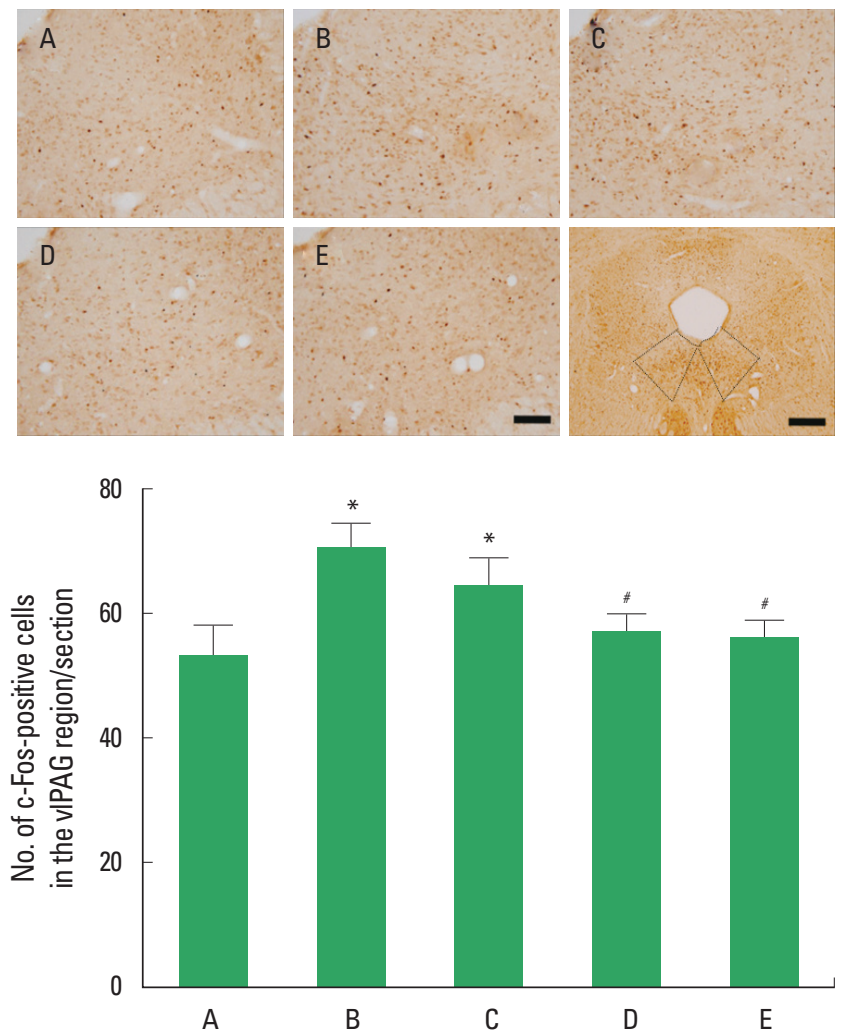

Fig. 3. Effect of electroacupuncture on c-Fos expression in the ventral lateral periaqueductal gray (vIPAG). Upper panel: Photomicrographs showing immunostaining for c-Fos-positive cells. The scale bar represents $100 \mu \mathrm{m}(\mathrm{A}-\mathrm{E})$ and $400 \mu \mathrm{m}$. Lower panel: Number of c-Fos positive cells in each group. A, sham-operation group; B, sciatic crushed nerve injury (SCI)-induced group; $\mathrm{C}$, SCl-induced and non-acupoint acupuncture group; D, SCl-induced and ST36-acupoint acupuncture group; E, SCl-induced and electroacupuncture group. ${ }^{*} P<0.05$ compared to the sham-operation group. ${ }^{\sharp} P<0.05$ compared to the SCl-induced group. 

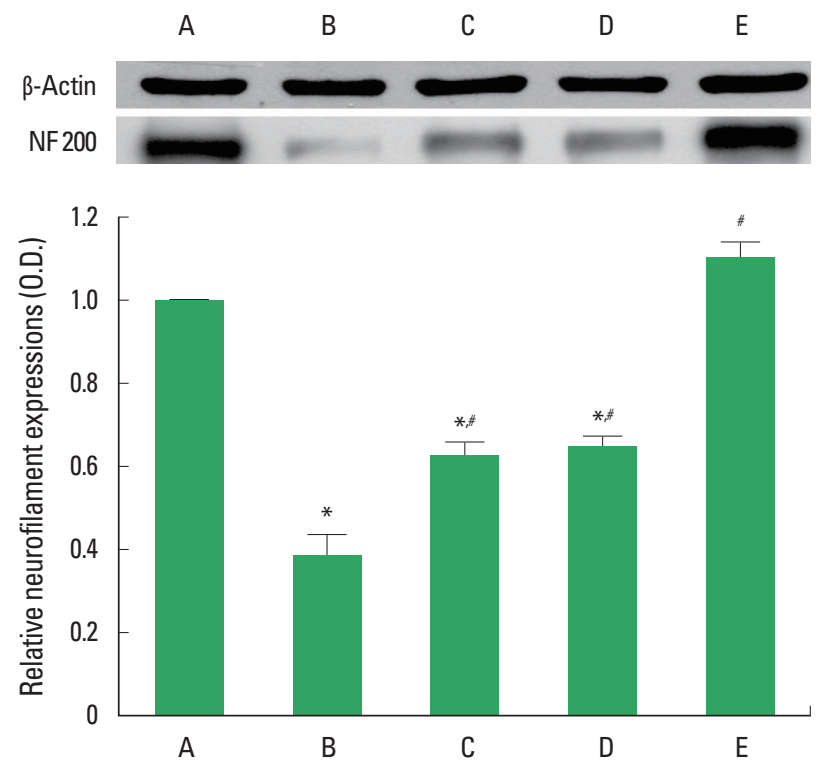

Fig. 4. Effect of electroacupuncture on neurofilament (NF)-200 expression in the sciatic nerve. Upper panel: Western blot analysis of NF-200 in the sciatic nerve. Lower panel: The mean optical density of NF-200 expression in each group. A, sham-operation group; $B$, sciatic crushed nerve injury (SCI)-induced group; C, SCl-induced and non-acupoint acupuncture group; D, SCl-induced and ST36-acupoint acupuncture group; E, SCl-induced and electroacupuncture group. ${ }^{*} P<0.05$ compared to the sham-operation group. ${ }^{\sharp} P<0.05$ compared to the SCl-induced group.

PVN was increased by SCI. Acupuncture at ST36-acupoint and EA treatment decreased the number of c-Fos-positive cells in the PVN of the SCI-induced rats.

\section{c-Fos expression in the vIPAG}

c-Fos expressions in the vlPAG are presented in Fig. 3. The present results showed that the number of c-Fos-positive cell in the vIPAG was increased by SCI. Acupuncture at ST36-acupoint and EA treatment decreased the number of c-Fos-positive cells in the vlPAG of the SCI-induced rats.

\section{NF-200 expression in the sciatic nerve}

NF-200 expressions in the sciatic nerve analyzed by western blotting are presented in Fig. 4. The present results showed that NF-200 expression in the sciatic nerve was decreased by SCI. Acupuncture and EA treatment increased NF-200 expression in the sciatic nerve of the SCI-induced rats. EA showed most potent increasing effect on NF-200 expression.

\section{BDNF expression in the sciatic nerve}

BDNF expressions in the sciatic nerve analyzed by western blotting are presented in Fig. 5. The present results showed that
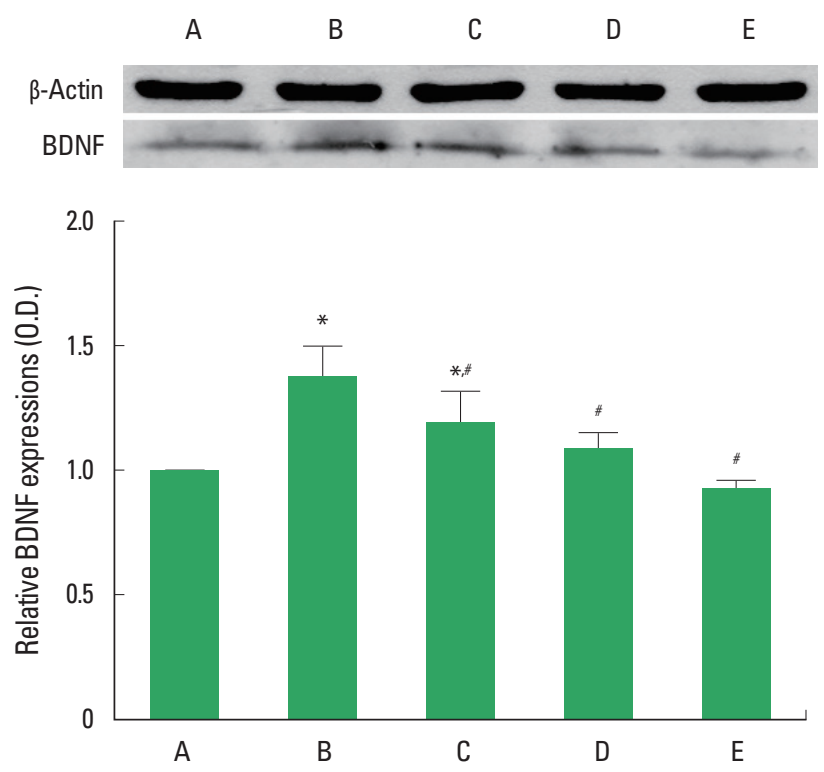

Fig. 5. Effect of electroacupuncture on brain-derived neurotrophic factor (BDNF) expression in the sciatic nerve. Upper panel: Western blot analysis of BDNF in the sciatic nerve. Lower panel: The mean optical density of BDNF expression in each group. A, sham-operation group; $B$, sciatic crushed nerve injury (SCI)-induced group; C, SCl-induced and non-acupoint acupuncture group; D, SCl-induced and ST36-acupoint acupuncture group; E, SCl-induced and electroacupuncture (EA) group. ${ }^{*} P<0.05$ compared to the sham-operation group. ${ }^{\#} P<0.05$ compared to the $S C l-$ induced group.

BDNF expression in the sciatic nerve was increased by SCI. Acupuncture and EA treatment decreased BDNF expression in the sciatic nerve of the SCI-induced rats. EA showed most potent deceasing effect on NF-200 expression.

\section{DISCUSSION}

The SFI is widely conducted to evaluate functional walking performance after peripheral nerve lesion, since it integrates sensory and motor fiber on specialized pattern of movement (Dinh et al., 2009). EA relieves the behavioral signs of neuropathic pain (Gim et al., 2011).

In the present study, SCI showed characteristic gait change with decrease of the SFI value. The rats in the EA-treated group showed increased SFI value. These results indicate that EA treatment improved locomotor function following SCI.

Nociceptive information reaches to the brain from the peripheral injury through ascending neuronal pathways (Vermeulen et al., 2014). c-Fos is commonly used to indicate activation of neurons in the brain induced by external inputs. c-Fos is used as a sensitive marker of the neural activation following sciatic nerve ligation (Nishimori et al., 2002). de Medeiros et al. (2003) demon- 
strated the EA exerted analgesic effect and influenced c-Fos expression in the rats with peripheral neuropathic pain. Increased c-Fos expression represents peripheral nerve activation (Han et al., 2017; Ko et al., 2016).

In the present study, c-Fos expression in the vlPAG and PVN was increased following SCI, while EA treatment suppressed SCI-induced c-Fos expression in the vIPAG and PVN. The present results indicate that neurons in the vIPAG and PVN were activated by SCI. In contrast, EA suppressed noxious stimulation on sciatic nerve, resulting in decrease of neuronal activation in the vlPAG and PVN.

Another important characteristic of nerve damage is NF activation. NF is the intermediate filament of never cells and NF is abundant in axonal process. NF is involved in the axonal transport and maintenance of cell shape as cytoskeletal components (Park et al., 2013), thus, NF activation is suppressed in the nerve system by various damages. Decreased immunoreactivity of NF-200 was observed in the rats with sciatic nerve ligation, in contrast, decrease of hypersensitivity increased axonal NF-200 immunoreactivity (Tomassoni et al., 2018).

In the present study, NF-200 expression in the sciatic nerve was decreased by SCI, suggesting that NF-200 expression was disturbed by hypersensitivity caused by nerve injury. In contrast, EA treatment increased NF-200 expression. The present results indicate that EA alleviated SCI-induced pain sensitivity, and then resulted in enhancing of NF expression.

Peripheral inflammation increased BDNF synthesis in the dorsal root ganglion, which was mediated by nerve growth factor and an elevated anterograde transport of BDNF to axon terminals in the spinal dorsal horn (Cho et al., 1997). Following nerve injury, the expression of BDNF in the superficial dorsal horn was increased (Kim et al., 2001). BDNF may play a critical role in synaptic plasticity in nociceptive signaling and regeneration (Kim et al., 2017; Sparg et al., 2004). Also Huang et al. (2004) reported EA accelerates nerve regeneration and functional recovery in delayed peripheral nerve injury in rats. BDNF is closely implicated in new neuronal generation (Hwang et al., 2016). However, the protective effects of neurotrophic factors on nerves have often controversy (Zhao et al., 2015). Lee et al. (2018) demonstrated that BDNF expression in the sciatic nerve was increased by SCI, in contrast, treatment with diosgenin suppressed BDNF expression.

In the present study, BDNF expression in the sciatic nerve was increased by SCI, suggesting that injury to the sciatic nerve induced BDNF expression for its regeneration. In contrast, EA treatment suppressed SCI-induced BDNF expression, suggesting that EA facilitated regeneration of injury to the sciatic nerve, thus over-expression of BDNF was suppressed.

In the present results, acupuncture and EA showed effectiveness on functional recovery from SCI. Of these, EA showed more potent effectiveness on functional recovery from SCI.

\section{CONFLICT OF INTEREST}

No potential conflict of interest relevant to this article was reported.

\section{ACKNOWLEDGMENTS}

This work was supported by 2009 research fund of Chungnam National University.

\section{REFERENCES}

Baek YH, Choi DY, Yang HI, Park DS. Analgesic effect of electroacupuncture on inflammatory pain in the rat model of collagen-induced arthritis: mediation by cholinergic and serotonergic receptors. Brain Res 2005; 1057:181-185.

Belevych N, Buchanan K, Chen Q, Bailey M, Quan N. Location-specific activation of the paraventricular nucleus of the hypothalamus by localized inflammation. Brain Behav Immun 2010;24:1137-1147.

Byun YH, Lee MH, Kim SS, Kim H, Chang HK, Lee TH, Jang MH, Shin MC, Shin MS, Kim CJ. Treadmill running promotes functional recovery and decreases brain-derived neurotrophic factor mRNA expression following sciatic crushed nerve injury in rats. J Sports Med Phys Fitness 2005;45:222-228.

Chang FC, Tsai HY, Yu MC, Yi PL, Lin JG. The central serotonergic system mediates the analgesic effect of electroacupuncture on ZUSANLI (ST36) acupoints. J Biomed Sci 2004;11:179-185.

Cherkin DC, Sherman KJ, Deyo RA, Shekelle PG. A review of the evidence for the effectiveness, safety, and cost of acupuncture, massage therapy, and spinal manipulation for back pain. Ann Intern Med 2003;138:898906.

Cho HJ, Kim JK, Zhou XF, Rush RA. Increased brain-derived neurotrophic factor immunoreactivity in rat dorsal root ganglia and spinal cord following peripheral inflammation. Brain Res 1997;764:269-272.

de Medeiros MA, Canteras NS, Suchecki D, Mello LE. Analgesia and c-Fos expression in the periaqueductal gray induced by electroacupuncture at the Zusanli point in rats. Brain Res 2003;973:196-204.

de Medinaceli L, Freed WJ, Wyatt RJ. An index of the functional condition of rat sciatic nerve based on measurements made from walking tracks. 
Exp Neurol 1982;77:634-643.

Dinh P, Hazel A, Palispis W, Suryadevara S, Gupta R. Functional assessment after sciatic nerve injury in a rat model. Microsurgery 2009;29: 644-649.

Dong ZQ, Ma F, Xie H, Wang YQ, Wu GC. Down-regulation of GFR $\alpha-1$ expression by antisense oligodeoxynucleotide attenuates electroacupuncture analgesia on heat hyperalgesia in a rat model of neuropathic pain. Brain Res Bull 2006;69:30-36.

Gim GT, Lee JH, Park E, Sung YH, Kim CJ, Hwang WW, Chu JP, Min BI. Electroacupuncture attenuates mechanical and warm allodynia through suppression of spinal glial activation in a rat model of neuropathic pain. Brain Res Bull 2011;86:403-411.

Han JH, Kim SE, Ko IG, Kim J, Kim KH. Afferent pathway-mediated effect of $\alpha 1$ adrenergic antagonist, tamsulosin, on the neurogenic bladder after spinal cord injury. Int Neurourol J 2017;21:178-188.

Huang C, Li HT, Shi YS, Han JS, Wan Y. Ketamine potentiates the effect of electroacupuncture on mechanical allodynia in a rat model of neuropathic pain. Neurosci Lett 2004;368:327-331.

Hwang DS, Kwak HB, Ko IG, Kim SE, Jin JJ, Ji ES, Choi HH, Kwon OY. Treadmill exercise improves memory function depending on circadian rhythm changes in mice. Int Neurourol J 2016;20(Suppl 2):S141-149.

Jung SY, Seo TB, Kim DY. Treadmill exercise facilitates recovery of locomotor function through axonal regeneration following spinal cord injury in rats. J Exerc Rehabil 2016;12:284-292.

Kim DS, Lee SJ, Cho HJ. Differential usage of multiple brain-derived neurotrophic factor promoter in rat dorsal root ganglia following peripheral nerve injuries and inflammation. Brain Res Mol Brain Res 2001;92: 167-171.

Kim YM, Seo TB, Kim CJ, Ji ES. Treadmill exercise with bone marrow stromal cells transplantation potentiates recovery of locomotor function after spinal cord injury in rats. J Exerc Rehabil 2017;13:273-278.

Ko IG, Moon BM, Kim SE, Jin JJ, Hwang L, Ji ES, Kim CJ, Kim TH, Choi $\mathrm{HH}$, Chung KJ. Effects of combination treatment of alpha 1-adrenergic receptor antagonists on voiding dysfunction: study on target organs in overactive bladder rats. Int Neurourol J 2016;20(Suppl 2):S150-158.

Kriz J, Zhu Q, Julien JP, Padjen AL. Electrophysiological properties of axons in mice lacking neurofilament subunit genes: disparity between conduction velocity and axon diameter in absence of NF-H. Brain Res 2000;885:32-44.
Lee BK, Kim CJ, Shin MS, Cho YS. Diosgenin improves functional recovery from sciatic crushed nerve injury in rats. J Exerc Rehabil 2018;14: 566-572.

Narita M, Ozaki S, Narita M, Ise Y, Yajima Y, Suzuki T. Change in the expression of c-fos in the rat brain following sciatic nerve ligation. Neurosci Lett 2003;352:231-233.

Nishimori T, Ikeda T, Terayama R, Ishida Y, Nakamura T, Otahara N. Effect of ionotropic glutamate receptor antagonists on Fos-like immunoreactivity in the dorsal horn following transection of the rat sciatic nerve. Brain Res 2002;934:81-86.

Park JH, Ko IG, Kim SE, Jin JJ, Hwang L, Kim CJ, Yoon SH, Hong J, Chung JY, Lee DW. Dexmedetomidine oral mucosa patch for sedation suppresses apoptosis in hippocampus of normal rats. Int Neurourol J 2017; 21(Suppl 1):S39-47.

Park JY, Jang SY, Shin YK, Suh DJ, Park HT. Calcium-dependent proteasome activation is required for axonal neurofilament degradation. Neural Regen Res 2013;8:3401-3409.

Price DD. Psychological and neural mechanisms of the affective dimension of pain. Science 2000;288:1769-1772.

Sallert M, Rantamäki T, Vesikansa A, Anthoni H, Harju K, Yli-Kauhaluoma J, Taira T, Castren E, Lauri SE. Brain-derived neurotrophic factor controls activity-dependent maturation of CA1 synapses by downregulating tonic activation of presynaptic kainate receptors. J Neurosci 2009;29:11294-11303.

Sparg SG, Light ME, van Staden J. Biological activities and distribution of plant saponins. J Ethnopharmacol 2004;94:219-243.

Tomassoni D, Di Cesare Mannelli L, Bramanti V, Ghelardini C, Amenta F, Pacini A. Treatment with acetyl-L-carnitine exerts a neuroprotective effect in the sciatic nerve following loose ligation: a functional and microanatomical study. Neural Regen Res 2018;13:692-698.

Vermeulen W, De Man JG, Pelckmans PA, De Winter BY. Neuroanatomy of lower gastrointestinal pain disorders. World J Gastroenterol 2014; 20:1005-1020.

Yacoubian TA, Lo DC. Truncated and full-length TrkB receptors regulate distinct modes of dendritic growth. Nat Neurosci 2000;3:342-349.

Zhao Q, Li ZY, Zhang ZP, Mo ZY, Chen SJ, Xiang SY, Zhang QS, Xue M. Polylactic-co-glycolic acid microspheres containing three neurotrophic factors promote sciatic nerve repair after injury. Neural Regen Res 2015;10:1491-1497. 